\title{
Giant Frontal Mucocele Occurring 32 Years after Frontal Bone Fracture: A Case Report
}

\author{
Yuuta Kamoshima $^{a}$ Shunsuke Terasaka $^{a}$ Yuji Nakamaru $^{b}$ \\ Dai Takagi ${ }^{b}$ Satoshi Fukuda ${ }^{b}$ Kiyohiro Houkin ${ }^{a}$ \\ Departments of a Neurosurgery and ${ }^{b}$ Otolaryngology and Head-Neck Surgery, \\ Graduate School of Medicine, Hokkaido University, Sapporo, Japan
}

\section{Key Words}

Giant $\cdot$ Frontal sinus $\cdot$ Mucocele $\cdot$ External approach

\begin{abstract}
Giant mucoceles of the frontal sinus are rare but their recognition is important in the differential diagnosis of proptosis and fronto-orbital lesions. The authors describe a patient with frontal giant mucocele with intracranial as well as orbit and ethmoid sinus involvement. Thirty-two years after a frontal sinus fracture, a 51-year-old female presented with headache, and left exophthalmos and ophthalmoplegia. Computed tomography and magnetic resonance imaging demonstrated a giant frontal sinus mucocele with extension into the left anterior cranial fossa. The mucocele was treated with a transcranial and endoscopic transnasal approach. The frontal sinus was then cranialized with reconstruction of the posterior wall, and finally a wide nasal drainage was performed. The clinical symptoms disappeared immediately after surgery.
\end{abstract}

\section{Introduction}

A paranasal mucocele is usually defined as an accumulation of mucosal secretions into a paranasal sinus with obstruction caused by inflammation, fibrosis, trauma, previous surgery, anatomical abnormality, or a mass lesion such as an osteoma [5]. When the mucocele becomes infected it is called a pyomucocele or pyocele, which contains pus partially or fully in cysts [6]. The clinical manifestation of mucoceles is benign in most cases $[5,6]$. Most mucoceles arise in the frontal and ethmoid sinuses, but intracranial extension is uncommon [3,5]. In this paper, we describe a case of a 
giant pyomucocele with extension into the anterior cranial fossa 32 years after a primary frontal sinus fracture due to a traffic accident.

\section{Case Report}

A 51-year-old woman presenting with left exophthalmos and ophthalmoplegia associated with large swelling over the left orbit and frontal region during the last three months was referred to the Department of Ophthalmology of Hokkaido University Hospital. She had a history of a motor vehicle accident that resulted in a frontal sinus fracture when she was 19 years old. At that time she underwent cranioplasty via bifrontal craniotomy. As a result of this accident, she had already lost visual acuity in her right eye. After admission to the Department of Ophthalmology, an orbital malignant tumor was suspected, and a local biopsy was performed via a left eyebrow incision. The content of the frontal sinus was not tumor but a purulent fluid collection. She was then referred to an otolaryngologist and a neurosurgeon at the same hospital. Neurological examination revealed bilateral anosmia. Her left visual acuity was still normal; however, upward ocular movements of the left eye were limited. Magnetic resonance imaging (MRI) on admission to the Department of Otolaryngology revealed a large mass in the left frontal intracranial region. The mass lesion severely compressed the left frontal lobe (fig. 1). The posterior margin of the frontal mass was partially surrounded by bony fragments on bone window computed tomography (CT). Bone window CT also demonstrated a defect of the posterior wall of the frontal sinus, where the mucocele wall attached to the dura mater, and thinning and inferior depression of the superior orbital roof. Her left orbit was displaced toward the posterior ethmoid sinus (fig. 2).

We planned for surgical excision of a giant frontal mucocele extending into the anterior cranial fossa. A single use of a Draf type III endoscopic frontal drill-out was technically difficult in this case as the orbit was so displaced that there was high risk for visual loss in her left eye [1]. A combined onestage external coronal and endoscopic transnasal approach were then performed. During surgery, we used the same coronal skin incision as the previous one and prepared a pericranial flap before the craniotomy. Except for the location just under the skin incision line, the pericranium was generally intact. A bifrontal trans-basal external table craniotomy was performed. Removal of the outer table of the frontal bone revealed a thin encapsulated cyst containing yellowish pus. While the posterior wall of the capsule was separated from the dura mater, adhesions containing bony fragments were noted between the dura mater and the capsule. After complete removal of the sinus mucosa, frontal sinus drainage (enlargement of the fronto-nasal duct) was achieved by a combined external trans-basal approach and endonasal endoscopic approach. The dura mater over the frontal base was intact and allowed to lie free in the cavity under the cover of the pericranial flap to shield between the epidural space and the paranasal sinus or the nose. Culture of the purulent fluid collection was negative. The postoperative course was uneventful, and all preoperative signs and symptoms resolved immediately after surgery. The patient has not shown a recurrence in six months.

\section{Discussion}

Frontal sinus mucoceles represent a relatively rare, slow-growing pathology. They are usually clinically silent and are caused by the loss of drainage properties of the sinus mucosa. They can have orbital, anterior cranial fossa, and intracranial involvement. Posttraumatic or postoperative mucoceles can appear several years after the original event. Mucoceles that result from trauma are mostly due to compromised ventilation, and can occur between 1 and 35 years later in the literature [4]. In our case, the mucocele developed 32 years after trauma and operation. This suggests the need for long-term follow-up after injury and conscientious anamnesis in suspicious cases. In our opinion, it is good practice to pay attention to post-trauma or postoperative patients with the possibility of mucocele occurring throughout their life. Frontal sinus mucocele is a disease that falls between the fields of otolaryngology and neurosurgery. 
Treatment requires removal of the mucocele sac and the offending obstruction with reestablishment of normal sinus drainage. Success rates ranging from 78 to $97 \%$ have been reported for external approaches, such as trephination, the Lynch and modified Lynch procedures, and osteoplastic flap techniques with or without frontal sinus obliteration [7]. Expansive growth of the frontal sinus mucocele may result in remodeling or destruction of the posterior wall of the frontal sinus. Increased intracranial pressure, due to mass effects of the mucocele, may result in a variety of neurological symptoms $[5,8]$. The most frequent symptoms of mucocele formation are frontal headache, proptosis, diplopia, nasal congestion, fluid leakage from the nose and swelling over the forehead [2]. A sinus mucocele presents as a non-enhancing, lowattenuation expansile mass on CT. MRI demonstrates mucoceles as well-defined, expansile masses with variable signal intensities on T1 and T2 images. These variations in signal intensities depend primarily on the state of hydration, protein content and viscosity of the content of mucocele [9]. In our patient, preoperative CT showed the destruction of the frontal sinus and remodeling of bone in the frontal sinus, and also revealed that the mucocele was directly attached to the dura mater. Operatively, the dura mater was very fragile at the points of contact with the mucocele. The safest way to treat a mucocele of this kind is to resect the sinus mucosa completely. This time we performed a standard external approach combined with cranialization of the frontal sinus (removal of posterior wall), removal of mucosa, and wide nasal drainage from the frontal base to the nasal cavity. We consider cranialization and reconstruction of nasofrontal duct through coronal access to be the most appropriate method when the posterior wall of the frontal sinus is remodeled. In most cases, extradural spaces which are formed after surgery become obliterated by the expansion of the frontal lobe within several weeks. In addition, vascularized local flaps are highly effective in preventing contamination of the anterior cranial fossa. For our patient, we chose a pericranial flap to shield from the paranasal space. However, when the mucocele is diagnosed in an early pre-invasive stage, the treatment options include minimally invasive surgery and endoscopic treatment, which have considerably less morbidity.

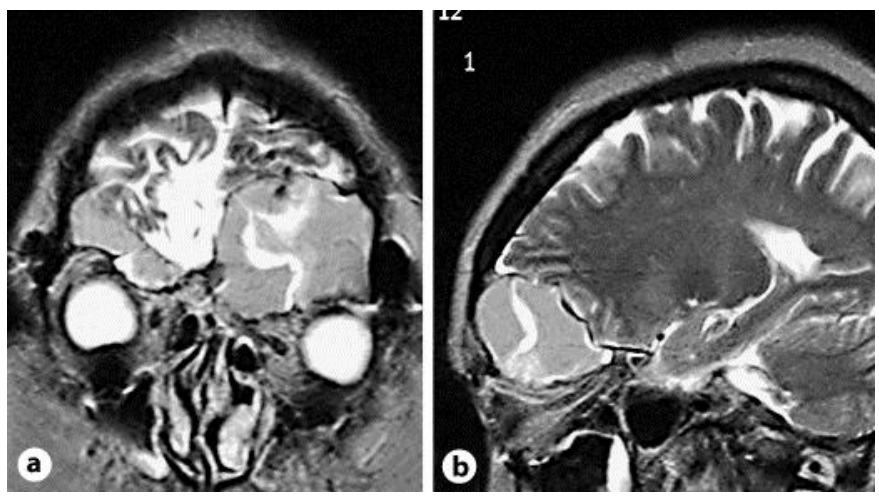

Fig. 1. Brain MRI revealed the left frontal mucocele with intracranial extension. a Coronal and b sagittal images (a, b: T2-weighted images). 

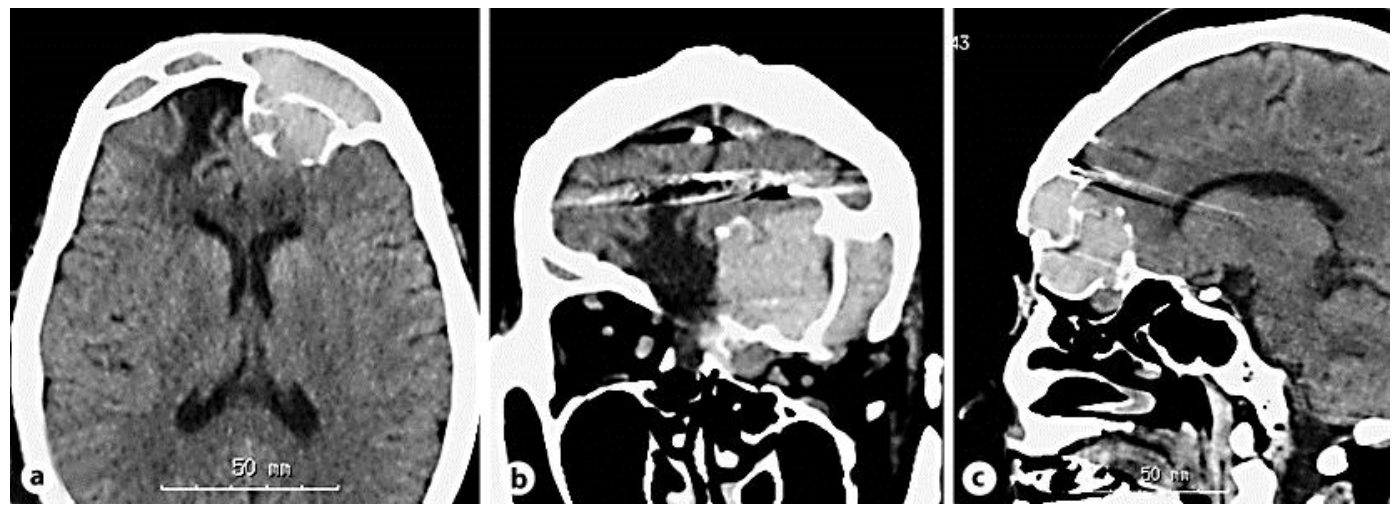

Fig. 2. Axial (a), coronal (b) and sagittal (c) CT scans demonstrated the giant frontal mucocele with extension into the left orbit, anterior ethmoid sinus and anterior cranial fossa with bone remodeling.

\section{References}

1 Anderson P, Sindwani R: Safety and efficacy of the endoscopic modified Lothrop procedure: a systematic review and meta-analysis. Laryngoscope 2009;119:1828-1833.

$\checkmark 2$ Constantinidis J, Steinhart H, Schwerdtfeger K, Zenk J, Iro H: Therapy of invasive mucoceles of the frontal sinus. Rhinology 2001;39:33-38.

-3 Kawaguchi S, Sakaki T, Okuno S, Ida Y, Nishi N: Giant frontal mucocele extending into the anterior cranial fossa. J Clin Neurosci 2002;9:86-89.

-4 Koudstaal MJ, van der Wal KG, Bijvoet HW, Vincent AJ, Poublon RM: Post-trauma mucocele formation in the frontal sinus; a rationale of follow-up. Int J Oral Maxillofac Surg 2004;33:751-754.

-5 Nakayama T, Mori K, Maeda M: Giant pyocele in the anterior intracranial fossa - case report. Neurol Med Chir (Tokyo) 1998;38:499-502.

-6 Stankiewicz JA, Newell DJ, Park AH: Complications of inflammatory diseases of the sinuses. Otolaryngol Clin North Am 1993;26:639-655.

7 Ulualp SO, Carlson TK, Toohill RJ: Osteoplastic flap versus modified endoscopic Lothrop procedure in patients with frontal sinus disease. Am J Rhinol 2000;14:21-26.

-8 Voegels RL, Balbani AP, Santos Júnior RC, Butugan 0: Frontoethmoidal mucocele with intracranial extension: a case report. Ear Nose Throat J 1998;77:117-120.

9 Weber R, Draf W, Keerl R, Kahle G, Schinzel S, Thomann S, Lawson W: Osteoplastic frontal sinus surgery with fat obliteration: technique and long-term results using magnetic resonance imaging in 82 operations. Laryngoscope 2000;110:1037-1044. 